This document is confidential and is proprietary to the American Chemical Society and its authors. Do not copy or disclose without written permission. If you have received this item in error, notify the sender and delete all copies.

\title{
Evolution of Graphene Molecules: Structural and Functional Complexity as Driving Force behind Nanoscience
}

\begin{tabular}{|r|l|}
\hline Journal: & ACS Nano \\
\hline Manuscript ID: & nn-2014-03283d \\
\hline Manuscript Type: & Perspective \\
\hline Date Submitted by the Author: & 17-Jun-2014 \\
\hline Complete List of Authors: & Müllen, Klaus; Max-Planck-Institute for Polymer Research, \\
\hline \multicolumn{2}{|l}{} \\
\hline
\end{tabular}

SCHOLARONE

Manuscripts 


\title{
Evolution of Graphene Molecules: Structural and Functional Complexity
} as Driving Forces behind Nanoscience

\author{
Klaus Müllen \\ Max-Planck-Institute for Polymer Research \\ Mainz, Germany
}

\begin{abstract}
The evolution of nanoscience is based on the ability of the fields of chemistry and physics to share competencies through mutually beneficial collaborations. With this in mind, in this Perspective, I describe three classes of compounds: rylene dyes, polyphenylene dendrimers, as well as nanographene molecules and graphene nanoribbons, which have provided a superb platform to nurture these relationships. The synthesis of these complex structures is demanding, but also rewarding because they stimulate unique investigations at the singlemolecule level by scanning tunneling microscopy and single-molecule spectroscopy. There are close functional and structural relationships between the molecules chosen. In particular, rylenes and nanographenes can be regarded as honeycombtype, discoid species composed of fused benzene rings. The benzene ring can thus be regarded as a universal modular building block. Polyphenylene dendrimers serve, first, as a scaffold for dyes en route to multichromophoric systems and, second, as chemical precursors for graphene synthesis. Through chemical design, it is possible to tune the properties of these systems at the single-molecule level and to achieve nanoscale control over their self-assembly to form multifunctional (nano)materials.
\end{abstract}

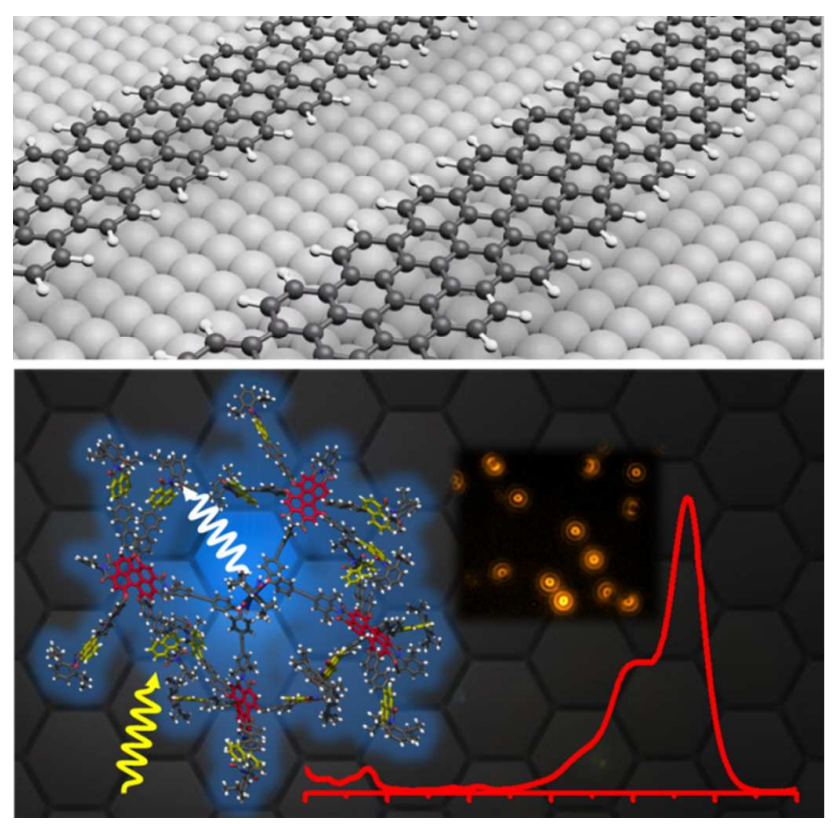


Chemistry is an essential part of nanoscience because it involves the materials, either molecules or particles, that make up the field, while also introducing an artistic element through eloquent synthetic design. Because nanoscience focuses on nanosized objects and their assemblies, it is imperative to acknowledge the need to visualize and to manipulate the individual molecular building blocks by physical methods such as scanning tunneling microscopy (STM) and single-molecule spectroscopy (SMS). Herein, I describe how synthetic chemistry and these physical characterization techniques have formed a beneficial, yet mutually challenging collaboration aimed at advancing nanoscience. ${ }^{1}$

However, more sophisticated characterization by these methods demands structurally perfect and increasingly complex, multifunctional molecules. For example, areas like electronics and optoelectronics require conjugated molecules with extended $\pi$-systems, ${ }^{2-5}$ which represent an enormous playground for the synthesis of both small organic molecules and polymers. The unique features of these structures, such as the ability to take up and to transport electrons or to interact with light and to undergo excitation energy transfer, is what qualifies them as active components of devices. By examining the field of nanoscience, one can conclude that the synthesis of new complex molecules has spurred new physical experiments capable of characterizing them, and, in turn, these new physical techniques have encouraged the pursuit of even more complicated syntheses. Therefore, I wish to demonstrate the advantages of the fruitful interplay between physics and chemistry for three classes of conjugated molecules: rylene-type chromophores (Scheme 1); shape-persistent dendrimers made from twisted, tightly packed interlocked benzene rings; and graphene molecules and related graphene nanoribbons (GNRs, Scheme 1).

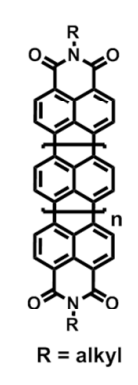

1

$1 \mathrm{a}(\mathrm{n}=0)$

$1 b(n=1)$

1c $(n=2)$

$1 d(n=3)$

$1 \mathrm{e}(\mathrm{n}=4)$

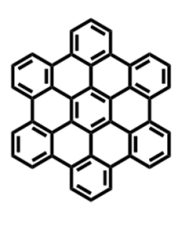

2

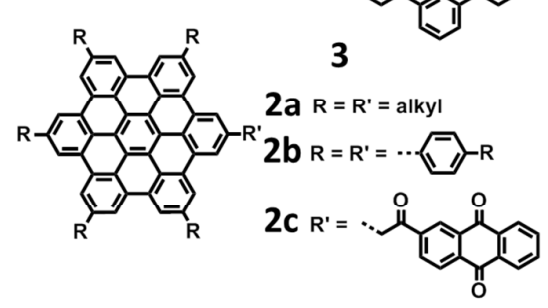

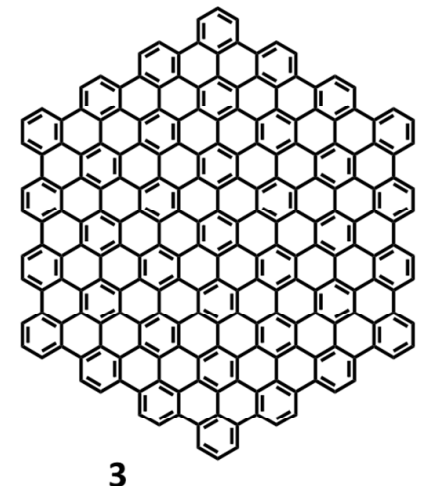

2a $R=R^{\prime}=$ alkyl

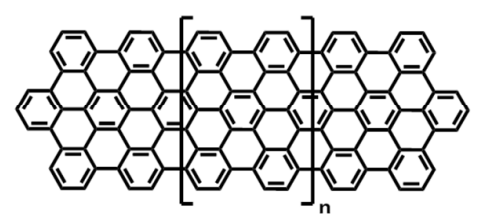

4

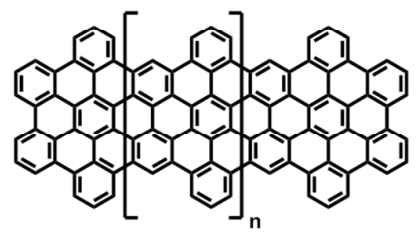

$4 a$

Scheme 1: Rylenes (1), graphene molecules (2) (3) and graphene nanoribbons (4) 
These examples of conjugated species were chosen based on a few obvious characteristics.

Chromophores, while representing a seemingly mature field of chemistry, are still in high demand from both an industrial and a fundamental point of view. One reason for this undiminished need for these materials is that chromophores can serve not only as colorants, but also as semiconductors, sensors, and biological probes. ${ }^{6}$ In regard to single-molecule properties, the workhorse of the SMS community has been the red terrylene dye (TDI, 1b). However, more complex studies have required chromophores with a broad variation of absorption and emission wavelengths and, even more important, ultrahigh photostability. ${ }^{7}$

Control over functional groups such as redox centers, catalysts, or receptors requires their perfect nano-site definition. This has prompted the synthesis of dendrimers as scaffolds for the design of "unimolecular" functional nanoparticles. ${ }^{8}$ Our polyphenylene dendrimers have provided such structures due to their shape persistence, which guarantees geometrically defined placement of the functional units in their nanoenvironments.

There has been a tremendous amount of effort spent on the fabrication of graphene and its derivatives through chemical and physical methods with varying levels of sophistication. ${ }^{9}$ Currently, graphenes are predominately obtained by top-down methods (i.e., exfoliation from graphite), ${ }^{10}$ but these methods yield products that lack structural perfection, especially when employing graphene oxide as an intermediate. Hence, it is necessary to utilize synthetic, bottom-up methods to achieve graphene materials with control over their chemical and physical structures. ${ }^{11-13}$

Herein, the benzene ring serves as a modular building block in proceeding from small organic molecules to extended oligomers and finally their related polymers. Next to the size of the chemical structure, the second most important design principle is their dimensionality. Linear (one-dimensional [1D]) poly-para-phenylenes are the prototype of conjugated polymers, with their two-dimensional (2D) analogues being graphene molecules, which we regard as 2D polymers. Remarkably enough, we synthesize them from dendritic three-dimensional (3D) polyphenylenes. It is this evolution of chemical synthesis that we define as a true bottom-up protocol.

Interdisciplinary efforts appear to be a key ingredient of nanoscience, since it is too difficult for a single research group to possess the necessary combination of chemical and physical expertise. Finding the right partners, however, can be another obstacle. Fortunately, with our collaborators, we have found a successful balance between achievements in chemistry and physics while still challenging one another toward even more difficult shared goals. I will mention these collaborations in the appropriate sections to highlight their accomplishments, and, additionally, I would like to dedicate this article to each and every one of them. 


\section{Rylene Chromophores}

Perylene tetracarboxdiimide (PDI, 1a) has been known for nearly a century and it has found extensive commercial use, while also serving as the workhorse of many electronic and optoelectronic investigations. A bulk heterojunction solar cell fabricated from a PDI as the electron acceptor and a hexabenzocoronene (HBC, $\mathbf{2 b}$ ) derivative as the electron donor has paved the way toward nanophase separation in solar cells with the formation of separate percolation pathways of holes and electrons. ${ }^{14}$ The photovoltaic activity in electron acceptor/electron donor blends based on nanoscale phase segregated PDI architectures and poly-3-hexyl-thiophene $(\mathrm{P} 3 \mathrm{HT})$ has been monitored for the first time at the nanoscale by Kelvin probe force microscopy (KPFM), in collaboration with Paolo Samori. ${ }^{15}$ The build-up of a homologous series of rylene dyes (Figure 1) has enabled a systematic variation of absorption and emission wavelengths reaching far into the near infrared. Since the new opportunities for device fabrication have been extensively described elsewhere, ${ }^{8}$ here, I focus on the role of these chromophores as stimuli for nanoscience.

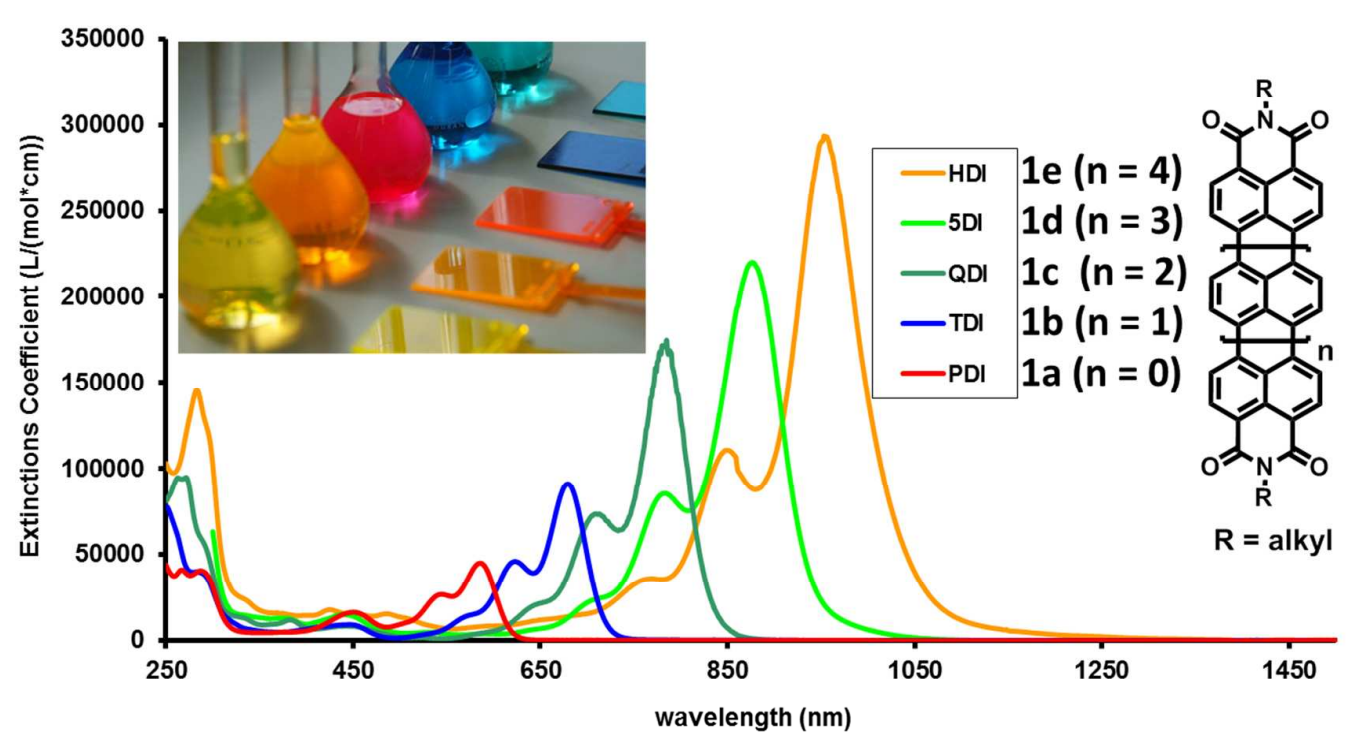

Figure 1: Homologous series of rylene dyes (1a-1e).

As expected, increases in the ribbon size up to $4 \mathrm{~nm}$ in length required the substitution of alkyl chains at the imide, as well as the ring positions to achieve soluble materials. Physisorption from solution and visualization of HDI (1e) by STM in the group of Steven De Feyter revealed the concentration-dependent formation of multilayers. Application of shear forces resulted in large-area alignment of these multilayers at the organic liquid-solid interface. Quinonediimine (QDI, 1c) was found to form two different patterns of $2 \mathrm{D}$ crystals, the relative abundance of which could also be controlled by shear flow. 7,16

As to optical detection, Christoph Bräuchle and Thomas Basché have shown terrylene diimide (TDI, 1b) to be an ideal chromophore for SMS due to its high 
fluorescence quantum yield and its emission above $700 \mathrm{~nm}$, which made it applicable as a tracking molecule for biological studies. ${ }^{7,17-19}$ The main advantage of TDI (1b) and its analogues over other dyes, some of which may be commercially available, is their extremely high photostability. This property also proved to be advantageous for the case of dibenzo-fused QDI, which has a bathochromically shifted emission spectrum. ${ }^{20,21}$ Here, SMS work by Nick van Hulst aimed to achieve coherent control and manipulation of vibrational wave-packet interference at room temperature, and these remarkable experiments required unprecedented stability of a chromophore made by design.

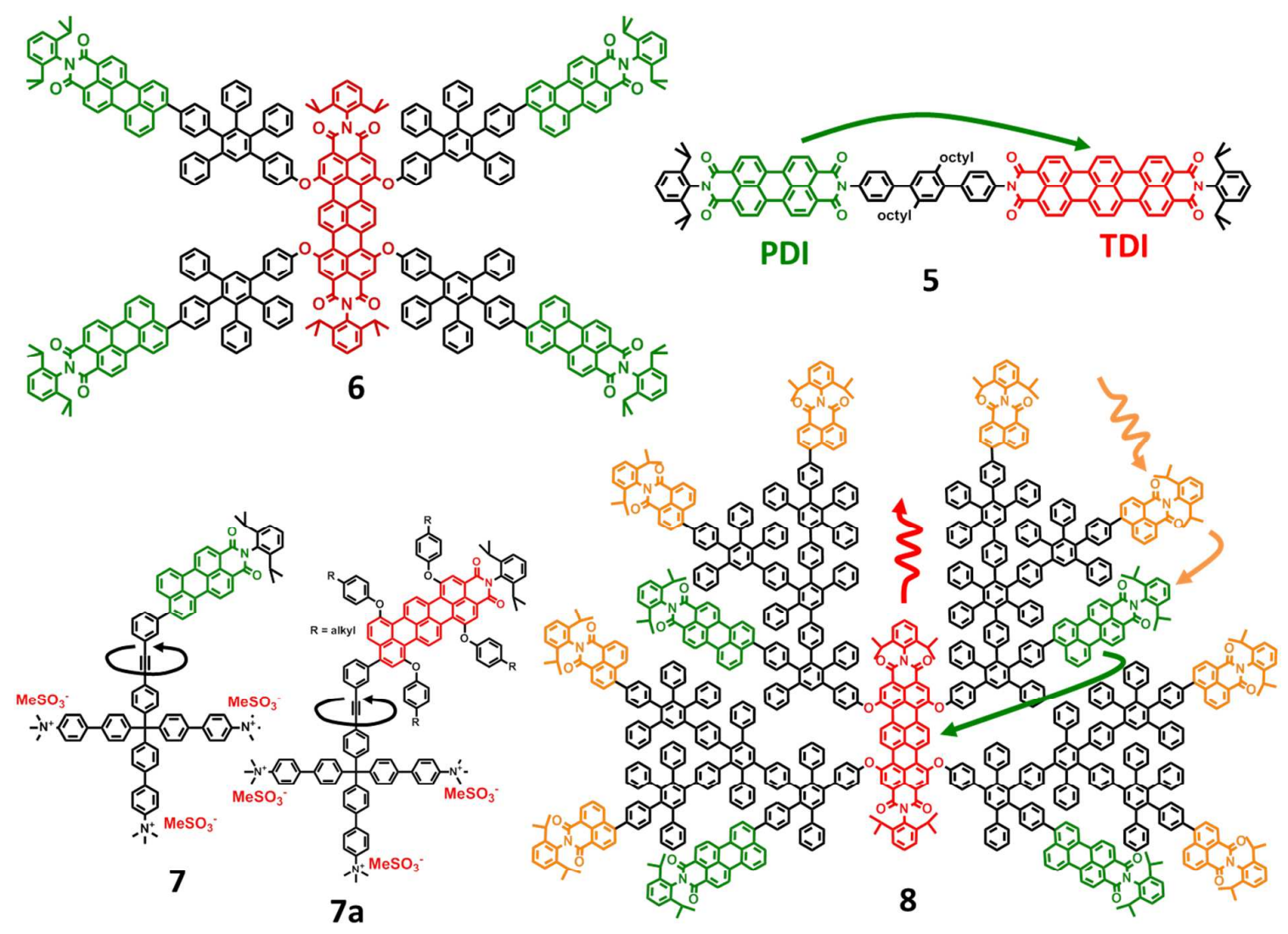

Scheme 2: Different multichromophoric systems.

The next logical step was to characterize multichromophores by SMS techniques (Scheme 2). Dyad 5 was synthesized with a PDI donor and TDI acceptor that were covalently connected by a rigid oligophenylene spacer, which was used to control the distance and dipole orientation factor between the units. Single-molecule spectroscopy provided unique insights into energy transfer mechanisms at the singlemolecule level, as well as to the limits of description by the Förster model. ${ }^{22}$ This process was further extended toward the modulation of energy transfer between individual molecules or the application of external stimuli, for example, by mechanical forces. Thus, together with the group of Thomas Basché, we made and studied multichromophore 6 in which a central TDI unit was surrounded by four perylene dicarboxy monoimide (PMI) dyes. ${ }^{23}$ This complex system was deposited on a surface 
and subjected to compressive stress exerted by the tip of an atomic force microscope. Conformational changes of the multichromophore occurred, leading to changes in the electronic states, which in turn were monitored by SMS. We could thereby detect spectral shifts that were reversible, as well as those that persisted, even after removal of the tip. A future challenge would be to incorporate structural functionalities that are capable of undergoing cis-trans isomerization (see below) into such chromophores synthetically, and to investigate the impact of this type of transformation at the molecular level by SMS.

Similarly complex in terms of synthesis and of photophysics, but equally rewarding from a nanoscience point of view, were the cases of molecules 7 and $7 a^{24}$ These materials were designed with the goal of resembling molecular machines. A perylene or terrylene dicarboxmonoimide moiety was attached to a tetraphenylmethane core yielding a tripod with three ammonium functional groups for anchoring onto a substrate surface. The dye was oriented either along the main $\mathrm{C}_{3}$ axis of the system or possessed a twist due to the presence of a meta-phenylene unit. The rotation of the dye around the central axis within the actual molecular rotor was monitored by defocused wide-field imaging in the group of Johan Hofkens. Here, it was a critical issue to bias the Brownian motion and to control the speed of the rotation, where one significant factor was the viscosity of the surrounding solvent. Another important factor, which was more difficult to understand, was the polarization of the excitation light field, because it would not be realistic to assume a sufficient optical torque. Rather, it was necessary to invoke a fluctuating friction model in this case. Thereby, the transition dipole tended to adopt the same direction as the optical field vector since this was when the probability for an excitation event was highest.

\section{Dendritic Multichromophores}

From there, we deemed it straightforward to proceed to more complex multichromophores by incorporating multiple dye molecules to investigate degenerate or directional energy transfer. In this case, it was necessary to achieve perfect spatial definition of the chromophores, and toward that end, we could involve a different object of our nanochemistry, the polyphenylene dendrimers. ${ }^{9}$ Scheme 3 illustrates their structures (9-11), which indeed represent a unique class amongst the dendrimer field because they i) are only made from twisted, tightly packed benzene rings, ii) can be obtained with structural perfection and monodispersity up to molecular weights above 1.5 MDa thanks to the use of repetitive Diels-Alder cycloaddition reactions, ${ }^{25}$ iii) possess outstanding chemical stability, and iv) are shape-persistent due to the lack of back-bending of the dendron arms. The semi-rigid character of these structures is a remarkable aspect that guarantees the perfect site definition of active groups such as chromophores, redox centers, or catalysts, and it is this feature that discriminates the polyphenylene macromolecules from other dendrimers. 


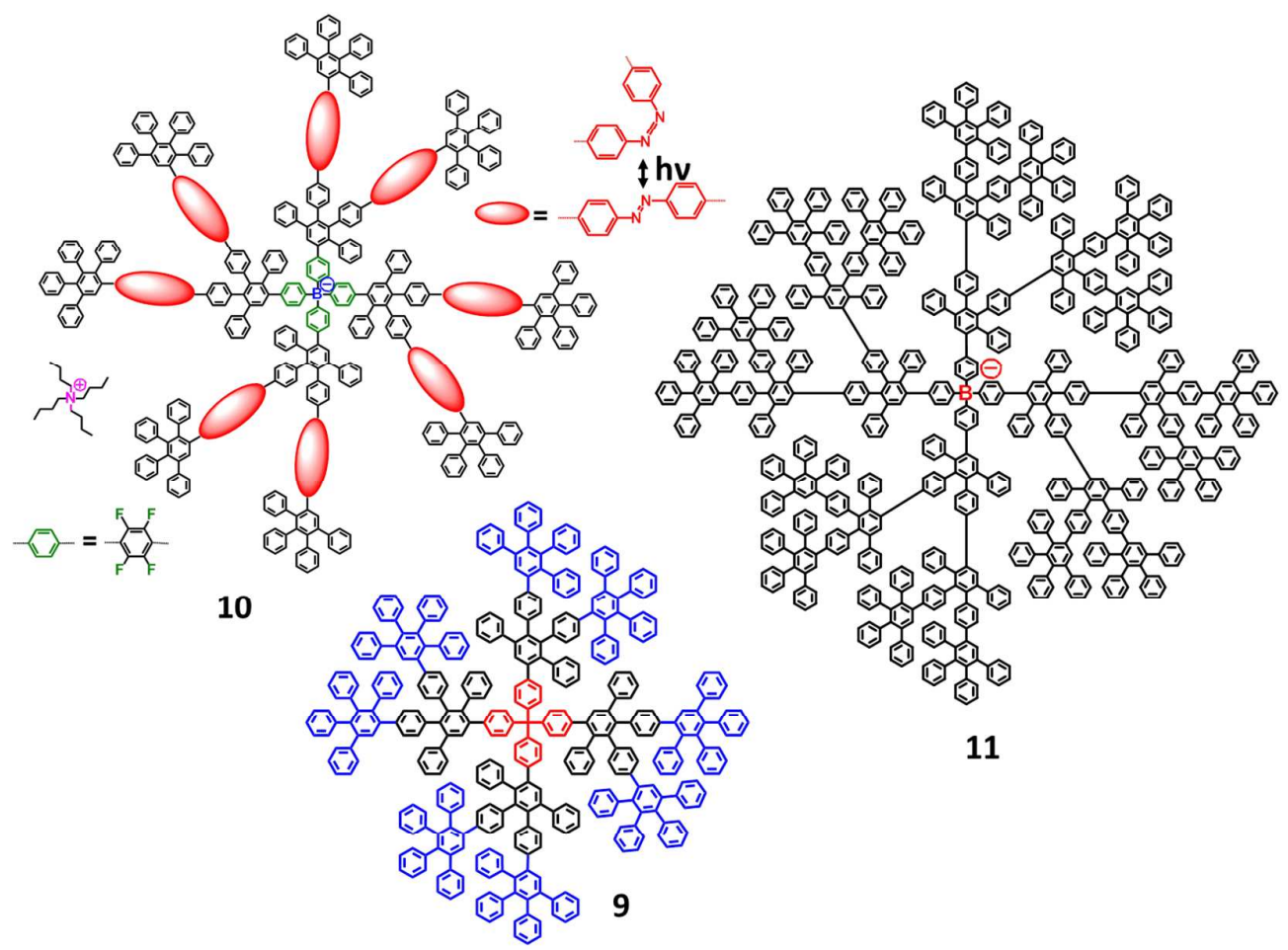

Scheme 3: Different polyphenylene dendrimers (only lower generation showed).

A special case was dendrimer $\mathbf{8}$, which possessed naphthalene dicarboxy monoimide antennae on the rim, PDI units throughout the scaffold, and a central TDI molecule as the core. ${ }^{26}$ This system generated a unique light-harvesting complex in which excitation energy transfer onto the core could occur in a direct or stepwise fashion, and this process was studied by Frans De Schryver and Johan Hofkens via SMS. Many other light-induced processes could also be analyzed through these functional 3D-macromolecules, such as electron transfer between different incorporated molecules or the isomerization of azobenzene units. The latter case is particularly appealing from a nanoscientific point of view since multiple azobenzene "hinges" placed in an otherwise rigid dendrimer scaffold (10) yielded spherical macromolecules whose size could be changed upon exposure to light. ${ }^{27}$ Furthermore, a unique aspect of these "breathing" dendrimers was that through the trans-cis isomerization of the azobenzenes the macromolecules could stably encapsulate guest species, which were only released upon opening the dendritic cages by the cis-trans transformation.

Thus, polyphenylene dendrimers create a unique functional nanoenvironment by way of a perfect covalent design. While these materials provide numerous opportunities that are appealing to nanoscience, they extend beyond the scope of the present article. However, I would be remiss not to mention some of the future challenges in the field: i) it has been shown that the inner voids of these dendrimers can be tuned to act as perfect receptors for explosives, and, in conjunction with an ultramicrobalance, they can be used as sensors with picomolar detection limits. ${ }^{28}$ It is important to utilize such host-guest interactions for other gas-sensing purposes; ii) 
borate functional groups can be encapsulated within the dendrimer core (11) to achieve weakly coordinating anions (WCAs). ${ }^{29}$ These macromolecular ions hold great promise for controlling chemical reactions, for example, in stabilizing cationic zirconocenium catalysts during polyolefin synthesis. On the physicochemical side, the dendritic shell can be used to separate spatially anionic and cationic species approaching the Bjerrum length of the ion pair. ${ }^{30}$

These dendrimers constructed from branched and densely packed benzene rings exhibit yet another feature that qualifies them as a key constituent of synthetically driven nanoscience: they serve as a molecular carbon source for transformation into $2 \mathrm{D}$ graphene molecules. The exciting potential of such a $3 \mathrm{D}$ to $2 \mathrm{D}$ interconversion is that it lays the groundwork for bottom-up synthesis of graphenes, and, more importantly, the connectivity of the dendrimer precursors defines the size and periphery of the graphenic products.

\section{Graphene Molecules and Graphene Nanoribbons}

Polycyclic aromatic hydrocarbons (PAHs), as pioneered by Erich Clar, have played a crucial role in the advancement of organic chemistry. ${ }^{31,32}$ In 1995 , we started a program toward obtaining larger and larger PAHs, with our "superbenzene" hexabenzocoronene (HBC, 2) as the most visible case. ${ }^{33}$ The synthesis included the planarization of the hexaphenylbenzene precursor 2' (Scheme 4). Furthermore, it was necessary to attach soft alkyl substituents to the parent HBC to make it solution processable. This enabled visualization by STM not only based upon vacuum deposition, but also upon physisorption on substrates such as highly oriented pyrolytic graphite (HOPG) from solution. Remarkably elegant STM experiments at the solid-liquid interface were then performed by Jürgen Rabe and Paolo Samori. ${ }^{1,34}$ Subsequent chemical developments have included i) increasing the size of the discs, often by designing homologous series; ii) varying the periphery from arm-chair to zigzag patterns; and iii) incorporating heteroatoms or forming ionic derivatives. In all cases, the synthesis proceeded via cyclodehydrogenation (flattening) of suitably designed polyphenylene precursors. ${ }^{35,36}$ Indeed, the precursor of nanographene 3 (C222) is compound 3', which turned out to be nothing more than a first-generation polyphenylene dendrimer with a hexaphenylbenzene core. In every case, the synergy between synthesis and STM studies proved to be of outstanding value. Additionally, STM was accompanied by scanning tunneling spectroscopy (STS) to gather information on the electronic structure of the surface-immobilized PAHs and to compare this information with the outcome of other spectroscopic characterization. ${ }^{37,38}$ 

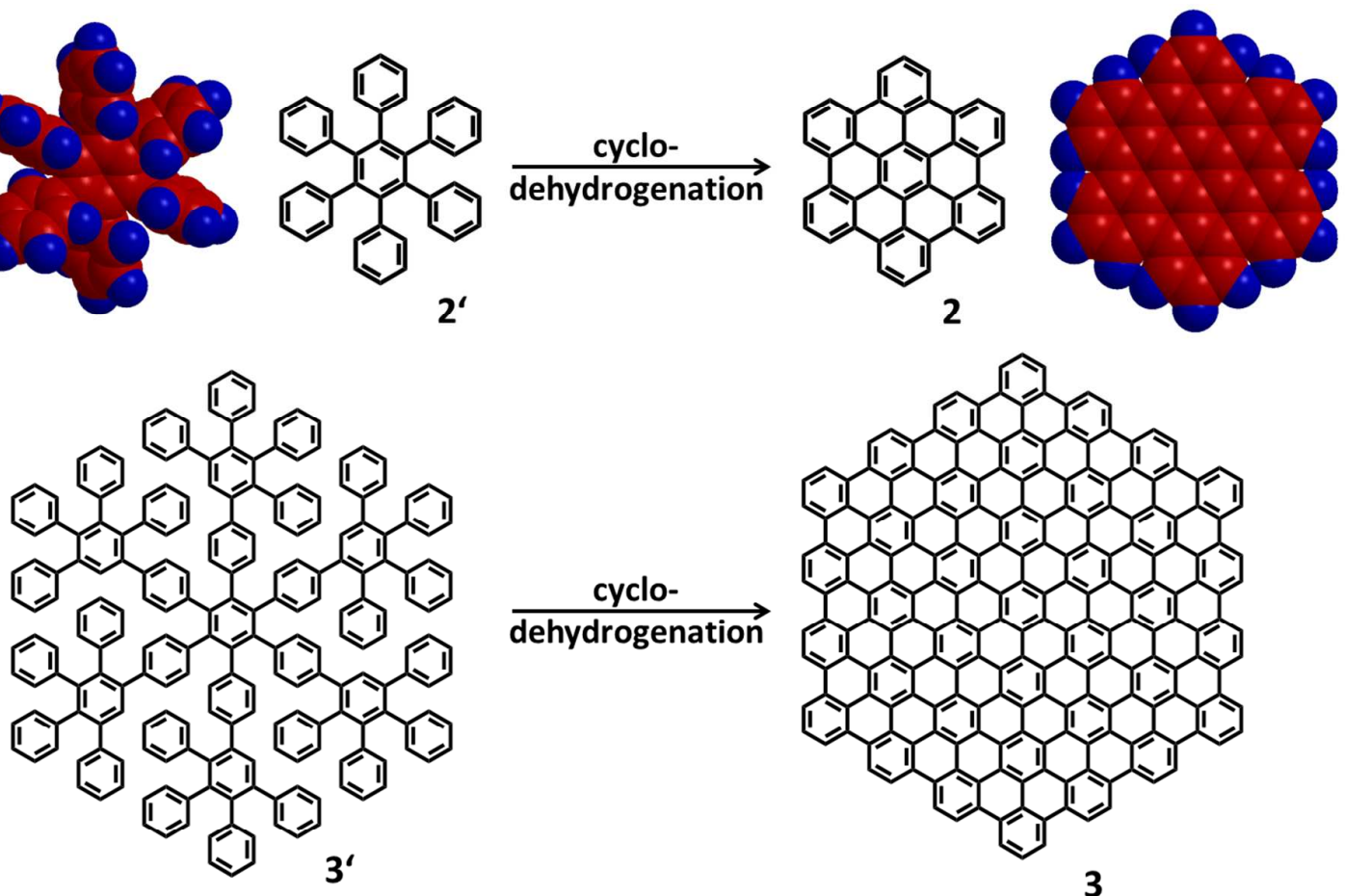

Scheme 4: From non-planar polyphenylene precursors to disc-type graphene molecules.

At this time, we would like to point out two more synthetic achievements that were particularly important from a nanoscience perspective. First, perfect edge chlorination of the discs induced ring puckering, and thus provided sufficient solubility for single crystal growth and structural analysis. ${ }^{39}$ These graphene molecules can be synthesized with diameters up to $4 \mathrm{~nm}$, and while this may be rather large from an organic chemistry stand point, they still possess structural perfection and are solution processable. It is possible to increase the size of these molecules even further; these materials will be outlined below for the case of graphene nanoribbons (GNRs).

Second, more complex PAH derivatives were made, such as the dyad $\mathbf{2 c}$ in which a HBC unit was connected to anthraquinone. ${ }^{40}$ After STM visualization, a donor molecule was placed from solution on top of the anthraquinone acceptor, thus creating a new dipole next to the HBC. The resulting electrical field could act as a gate for the tunneling current through the $\mathrm{HBC}$ and therefore afford a model case for a single-molecule field-effect transistor (FET). In other studies, HBC could be functionalized with two thiol anchor groups and then subjected to break junction-STM measurements of the electrical conduction. ${ }^{41}$ Molecular wires have been a longlasting query of nanoscience and a comparison of $1 \mathrm{D}$ and $2 \mathrm{D}$ species is among the key topics.

There is, admittedly, an appreciable gap between nanometer-sized, molecularly defined graphenes ${ }^{42}$ and the micron-sized graphene flakes made by top-down methods. Before we start to close this gap synthetically, let us take a brief look at the 
high charge carrier mobility for graphene, ${ }^{43,44}$ which would qualify graphene as a useful semiconductor in a high-modulation FET. ${ }^{45}$ However, this is hampered by the vanishing band gap of graphene, which excludes a proper off-behavior and limits the general use of graphene in digital electronics. Yet it is possible to open the band gap through geometric confinement, which has been demonstrated through the production of GNRs. ${ }^{46}$ With this in mind, various approaches toward the fabrication of GNRs have been taken, such as lithography of graphene precursors or unzipping of carbon nanotubes. ${ }^{14,47}$ The obvious disadvantage of such protocols is the lack of structural definition of the edges and this defines a pressing need for a bottom-up synthesis method.

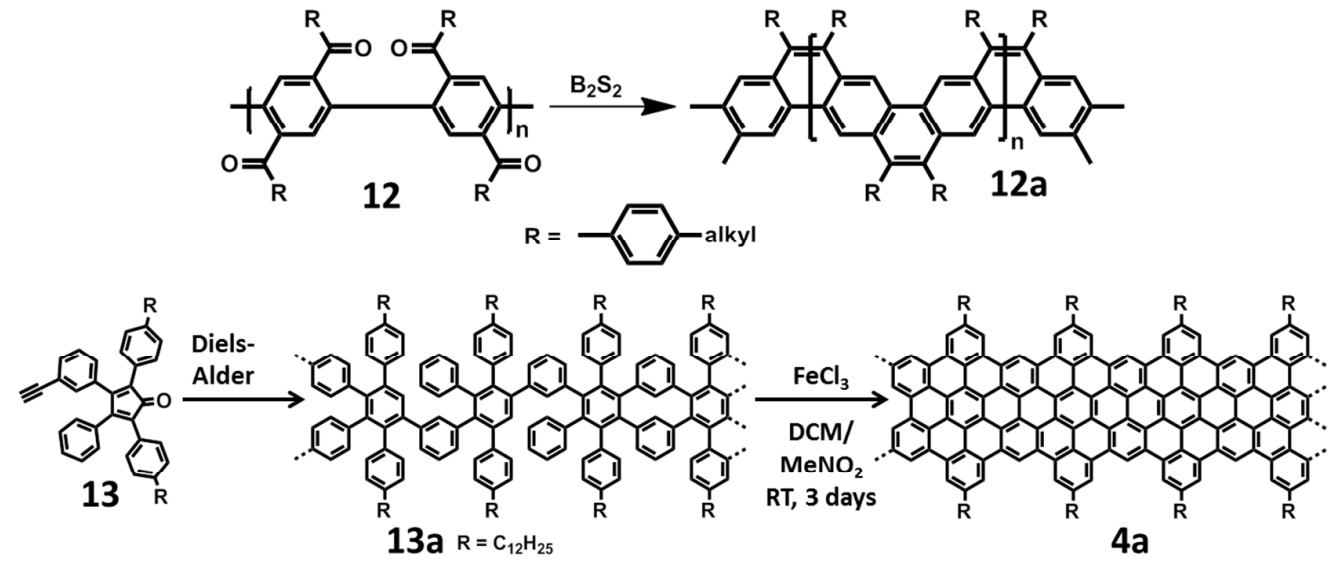

Scheme 5: Synthesis of graphene nanoribbons in solution.

It might be mentioned that as early as 1993 we synthesized polymer $12^{48}$ as part of our work toward double-stranded polyphenylenes, and $12 a$ is indeed a GNR (Scheme 5). Since 2010, we have introduced a whole new class of GNRs with polymer $4 a$ as the most recent example ${ }^{49}$ (Scheme 5). The synthesis included the twisted precursor polymer 13a which was obtained by a repetitive Diels-Alder cycloaddition of the AB-type monomer 13. The polymer was then, as described above, subjected to intramolecular dehydrogenation, yielding GNR 4a. The planarized macromolecule, due to its extensive alkyl substitution, was still solution processable, and after fractionation, it could be obtained with a length of $600 \mathrm{~nm}$. Visualization and proof of the structural perfection were achieved by the group of Steven De Feyter via STM after deposition onto graphite or a gold surface. Currentpotential curves were measured by Chongwu Zhou after the GNRs were deposited from solution between electrodes, and the resulting devices could even be used for gas sensing. ${ }^{50}$ Since the fabrication of FETs with organic semiconductors ${ }^{11,51}$ is often obstructed by large contact resistances it was important that the charge carrier mobilities could also be detected in a contact-free mode by terahertz spectroscopy in the group of Mischa Bonn. ${ }^{52}$

Some key challenges for synthesis of future chemical GNRs are: i) to further increase their size while also varying the aspect ratios, and control the edge structures; ii) to 
incorporate heteroatoms; iii) to form block copolymers from GNR blocks with different widths and thus different bandgaps; and iv) to end-cap with functional groups toward anchoring at electrodes. Needless to say, all of these structural changes are essential for future device studies, but require sophisticated chemical expertise.
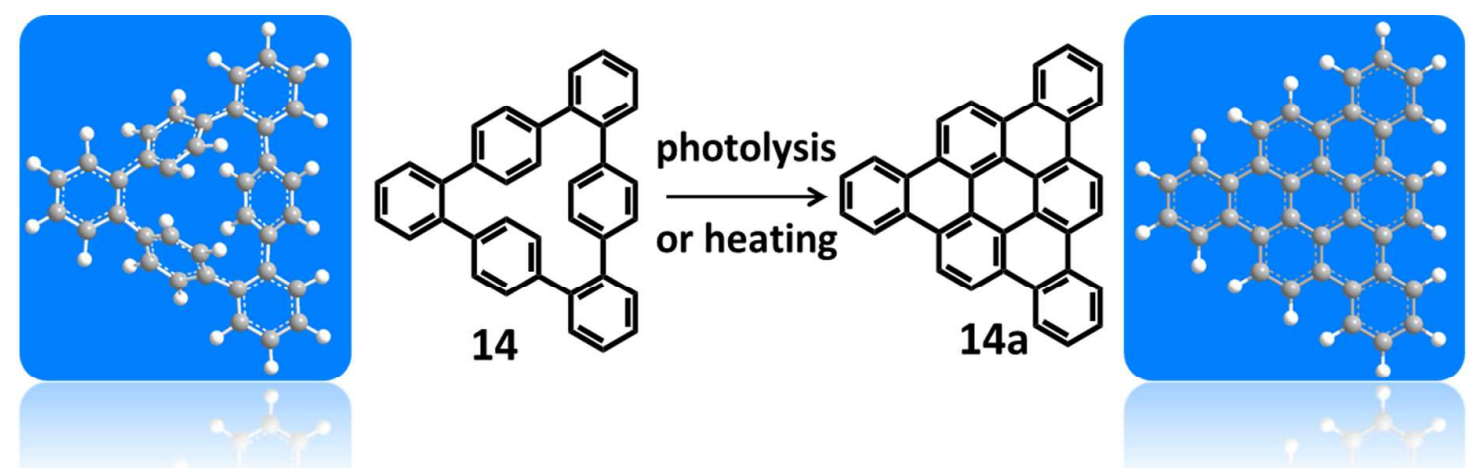

Scheme 6: Synthesis of tribenzocoronene 14a by electrocyclic ring closure from cyclophane 14.

These syntheses, while enabling rewarding nanoscience, will push the limits of classical polymer chemistry. However, there is one problem: the decreasing solubility of larger and larger ribbons. While the use of soft-landing in collaboration with Paolo Samori ${ }^{53}$ was shown to be a method to process larger and larger PAHs into ordered crystalline monolayers on a conductive substrate, such a deposition method is extremely slow. In this framework, a key breakthrough in view of processing limitations was pioneered by the group of Roman Fasel through an atomically precise GNR synthesis. Their protocol comprised UHV-deposition of small building blocks onto metal surfaces and monitoring of the whole process by STM. Our organic model reaction was the transformation of cyclophane 14 into the PAH 14a (Scheme 6). This stepwise electrocyclic ring closure could be accomplished by photolysis in solution, but also by heating the precursor on a $\mathrm{Cu}(111)$ surface with STM control. ${ }^{54}$ Related work in the literature ${ }^{55,56}$ made use of dibromo derivatives of aromatic building blocks that were immobilized on metal surfaces. Thermal carbon-halogen bond cleavage furnished intermediate diradicals that could still diffuse on the surface and polymerize to form conjugated polymer chains. In a similar fashion, we could achieve the polymerization of the dibromobianthryl building block 15a in spite of the prevailing steric hindrance. However, the formation of GNR 15 required subsequent flattening of the precursor polymer, and this became possible in a further heating step (Figure 2). ${ }^{57}$ Then, it was logical to apply a surface-bound monolayer synthesis to other polymer topologies such as chevron structures, ${ }^{57}$ which could simply be realized by the choice of the dibromo monomer. Furthermore, polymer networks became accessible by introducing tribromo-substituted building blocks. Along these lines, we could accomplish the synthesis of $2 \mathrm{D}$ layers with atomically precise pores for possible use as membranes. ${ }^{58}$ 

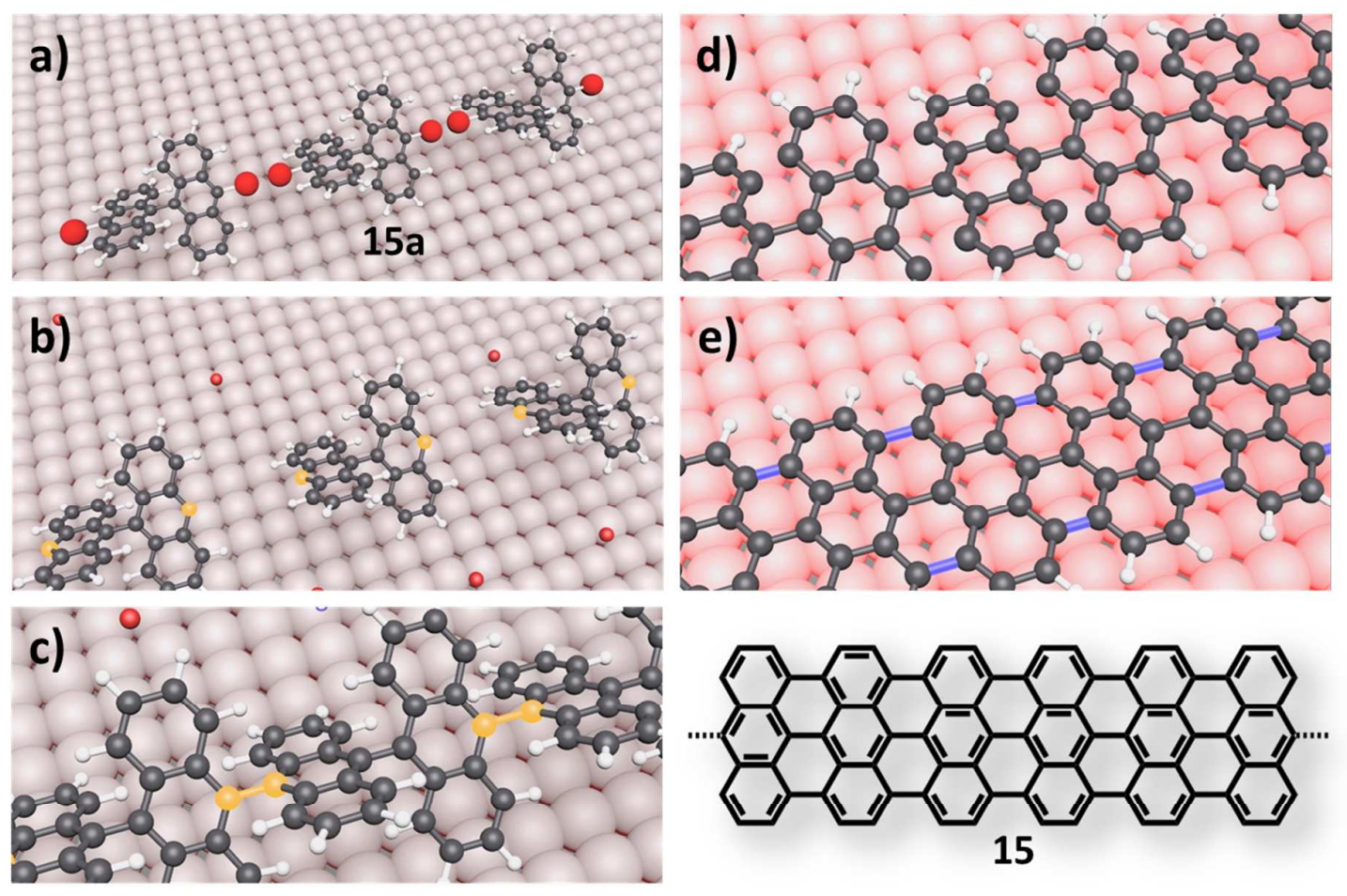

Figure 2: Schematic illustration for the surface-assisted synthesis of $N=7$ GNR 15. a) Vacuum deposition of 10,10'-dibromo-9,9'-bianthryl monomer 15a on a metal surface. b) Debromination of 15a to form surface-stabilized biradical intermediates. c) Radical polymerization to form covalent bonds between the monomer units. d) Preplanarization upon thermal activation, followed by e) surface-assisted cyclodehydrogenation to give GNR 15.

Chemical reactions on surfaces and, in particular, GNR synthesis under the control of STM and STS have become a very active new field of nanoscience. ${ }^{59-61}$ Many questions remain to be answered though, such as, i) the occurrence of side reactions or the coupling of neighboring ribbons, ii) the mechanism of polymer growth upon diffusion of intermediate oligomers, and iii) the feasibility of reactions other than arylaryl coupling. ${ }^{62,63}$ However, there are remarkable opportunities that mainly follow from the choice of the monomeric starting compound and that help to tailor the electronic structure of the product. This can be achieved, as with solution synthesis, via doping of the resulting GNR by incorporation of heteroatoms into the monomer or by varying the width and edges of the ribbon. Particularly exciting will be GNRs with zig-zag peripheries because they promise unique edge states, and thus offer an entry into spintronics. Another problem is the need to execute this synthesis from metal substrates. The latter enables STM control and might lead to the stabilization of reactive intermediates. Furthermore, they offer additional opportunities by preorganization of monomers or polymers on the surface or by combining different building blocks in a programmable fashion. However, conducting surfaces exclude charge transport measurements, and subsequent device fabrication would require transfer to insulating surfaces by lift-off techniques. 
From a physical point of view, we have looked at the characterization of graphene molecules by STM and SMS side by side since they both provide significant breakthroughs in single-molecule detection. Apart from the physical methods, there is also a structural connection between our families of graphene and rylene molecules. Indeed, rylene dyes represent a special topology of PAHs in which the HOMO-LUMO gaps are particularly small. Rylenes can also be regarded as a narrow GNR, and this relation could be further corroborated by a chemical reaction, the Hundsdiecker decarboxylation. ${ }^{64}$ Thereby, PDIs were transformed into tetrabromo perylenes 16 (Scheme 7). These compounds were used by Lifeng Chi and Harald Fuchs as unique building blocks for GNR 16a formation on gold surfaces. The same GNRs could also be achieved when starting from a tetrabromonaphthalene 17. A key aspect of both protocols is that ribbon formation does not require a cyclodehydrogenation step. It is mechanistically remarkable that the fusion of two naphthalene units via their peripositions proceeds by an aurate complex 17a in which the four peri-positions are connected by two gold centers.

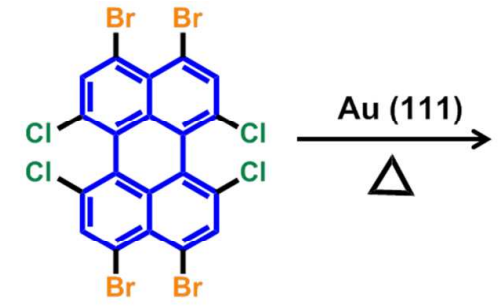

16

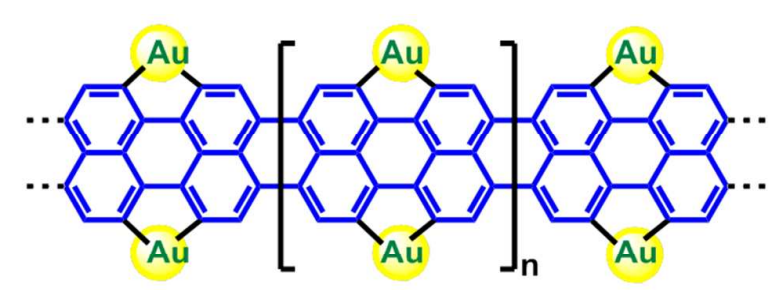

$16 a$
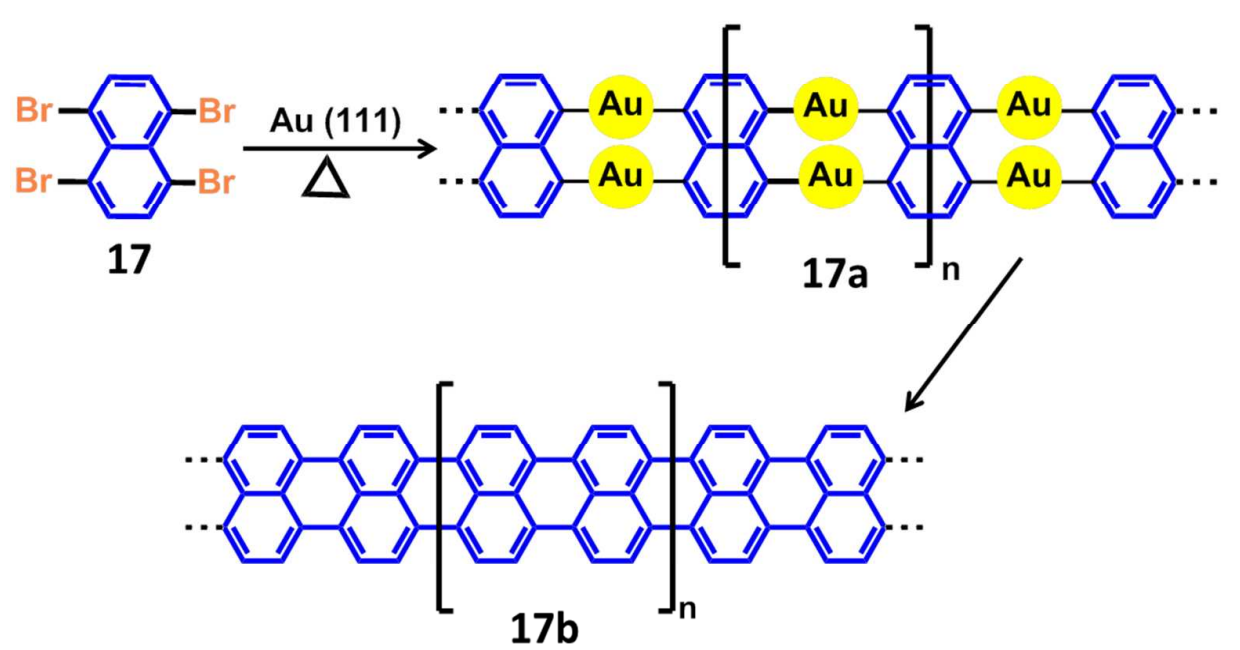

Scheme 7: On-surface synthesis of rylene-type graphene nanoribbons.

Therefore, it is a unique feature of our GNR fabrication, both in solution and on surfaces, that their geometric and electronic structures can be tuned by the nature of the monomeric building blocks. Some chemical challenges have been outlined already but many more can be envisaged, such as the inclusion of monodisperse oligomers as model compounds or selective reactions at the edges to tune the band 
gaps further. More physical methods would have to be employed, such as Raman spectroscopy and X-ray photoelectron spectroscopy, to complement these syntheses.

\section{Conclusion and Prospects}

The present cases were chosen to document the cross-fertilization between design and synthesis on one hand and single-molecule detection on the other. These selected examples should provide evidence that nanoscience must go beyond the catalogue of commercial compounds to make use of our collective chemical imagination. The toolbox of synthetic methods must be combined with overarching design concepts, where the size and dimensionality of molecules is of particular interest. The resulting bottom-up approach of materials synthesis offers the advantages of structural perfection and reproducibility.

While many further challenges have already been outlined, there remain no limits for the multidisciplinary interactions discussed herein. The beauty of materials synthesis cannot be further exemplified, although the many obstacles of extremely intricate syntheses have not been expounded on in this Perspective. It becomes obvious, however, that all these structural and functional modifications immediately create opportunities for fresh nanoscience, both in bulk and at the single-molecule level. While the emphasis of this Perspective has been on fundamental aspects, there is also room for applications of which a few cases shall be mentioned: rylenes can be used as near-infrared absorbers in heat management; surface-functionalized dendrimers act as drug-delivery vehicles capable of crossing the blood-brain barrier; detection of lung cancer from breath analysis requires chemiresistors based upon graphene molecules and carbon nanotubes; 1,2-diketone functionalities at the edge of graphenes establish catalytic activity in the dehydrogenation of ethylbenzene to styrene; structurally perfect graphene molecules hold special promise as semiconductors for FETs; and there is so much more to come.

\section{Acknowledgments}

The author acknowledges, next to the many partner groups, the dedicated work of many associates. Without their skill and creativity this Perspective would not have been possible. Also acknowledged is financial support by the EU projects FP7Energy-2010-FET Project Molesol (FP7-Energy-2010 256617), EU Project GENIUS (ITN-264694) Superior (ITN-238177), and the Integrated projects RADSAS (NMP3CT-2004-001561), ONE-P (NMP3-LA-2008-212311), and NAIMO (NMP4-CT-2004500355). Of particular importance is the ERC-Adv.-Grant 267160 (NANOGRAPH). Additional financial support was provided by Volkswagen Foundation, Max-PlanckSociety (ENERCHEM), Federal Ministry of Education and Research (BMBF) (Projects LiBZ and Graphenoid-Lagen), and the German Science Foundation (DFG) within the frame of the ESF Projects GOSPEL (09-EuroGRAPHENE-FP-001), SONS2-SUPRAMATES, DFG Priority Programs SPP 1355, SPP 1459, and the 
collaborative research project SFB 625. Industrial collaborations with BASF AG, Ludwigshafen were also essential to the success of this work.

\section{References}

1 Samori, P.; Rabe, J. P. Scanning Probe Microscopy Explorations on Conjugated (Macro)molecular Architectures for Molecular Electronics. J. Phys. Condens. Mater 2002, 14, 9955-9973.

2 Chua, L. L.; Zaumseil, J.; Chang, J. F.; Ou, E. C. W.; Ho, P. K. H.; Sirringhaus, H.; Friend, R. H. General Observation of $n$-Type Field-Effect Behaviour in Organic Semiconductors. Nature 2005, 434, 194--199.

3 Brédas, J. L.; Silbey, R. Conjugated Polymers. Kluwer Academic Publishers, The Netherlands 1991.

4. Henson, Z. B.; Müllen, K.; Bazan, G. C. Seeking Design Strategies for Organic Semiconductors beyond the Molecular Formula, Nat. Chem. 2012, 4, 699-704.

5 Diez-Perez, I.; Hihath, J.; Hines, T.; Wang, Z. S.; Zhou, G.; Müllen, K.; Tao, N. J. Controlling Single-Molecule Conductance Through Lateral Coupling of $\pi$ Orbitals. Nat. Nanotechnol. 2011, 6, 226-231.

6 Chen, L.; Li, C.; Müllen, K. Beyond Perylene Diimides: Synthesis, Assembly and Function of Higher Rylene Chromophores. J. Mater. Chem. C 2014, 2, 19381956.

7 Weil, T.; Vosch, T.; Hofkens, J.; Peneva, K.; Müllen, K. The Rylene Colorant Family-Tailored Nanoemitters for Photonics Research and Applications. Angew. Chem. Int. Ed. 2010, 49, 9068-9093.

8 Türp, D.; Nguyen, T. T. T.; Baumgarten, M.; Müllen, K. Uniquely Versatile: NanoSite Defined Materials Based on Polyphenylene Dendrimers. New J. Chem. 2012, 36, 282- 298.

9 Geim, A. Graphene: Status and Prospects, Science 2009, 324, 1530-1534.

10 Li, X.; Wang, X.; Zhang, L.; Lee, S.; Dai, H. Chemically Derived, Ultrasmooth Graphene Nanoribbon Semiconductors. Science 2008, 319, 1229-1232.

11 Dreyer, D. R.; Ruoff, R. S.; Bielawski, C. W. From Conception to Realization: An Historical Account of Graphene and Some Perspectives for Its Future. Angew. Chem. Int. Ed. 2010, 49, 9336-9344.

12 Zhu, Y. W.; Murali, S.; Cai, W. W.; Li, X. S.; Suk, J. W.; Potts, J. R.; Ruoff, R. S. Graphene and Graphene Oxide: Synthesis, Properties, and Applications. Adv. Mater. 2010, 22, 5226-5226.

13 Chen, L.; Hernandez, Y.; Feng, X. L.; Müllen, K. From Nanographene and Graphene Nanoribbons to Graphene Sheets: Chemical Synthesis. Angew. Chem. Int. Ed. 2012, 51, 7640-7654.

14 Schmidt-Mende, L.; Fechtenkötter, A.; Müllen, K.; Moons, E.; Friend, R. H.; MacKenzie, J. D. Self-Organized Discotic Liquid Crystals for High-Efficiency Organic Photovoltaics. Science 2001, 293, 1119-1122.

15 Liscio, A.; Palermo, V.; Fenwick, O.; Braun, S.; Müllen, K.; Fahlman, M.; Cacialli, F.; Samori, P. Local Surface Potential of pi-Conjugated Nanostructures by Kelvin Probe Force Microscopy: Effect of the Sampling Depth. Small 2011, 7, 634-639.

16 Yuan, Z.; Lee, S.-L.; Chen, L.; Li, C.; Mali, K. S.; De Feyter, S.; Müllen, K. Processable Rylene Diimide Dyes up to $4 \mathrm{~nm}$ in Length: Synthesis and STM Visualization. Chem. Eur. J. 2013, 19, 11842-11846. 
17 Jung, C.; Ruthardt, N.; Lewis, R.; Michaelis, J.; Sodeik, B.; Nolde, F.; Peneva, K.; Müllen, K.; Bräuchle, C. Photophysics of New Water-Soluble Terrylenediimide Derivatives and Applications in Biology, Chem. Phys. Chem. 2009, 10, 180-190.

18 Mais, S.; Tittel, J.; Basché, T.; Bräuchle, C.; Gohde, W.; Fuchs, H.; Müller, G.; Müllen, K. K Terrylenediimide: A Novel Fluorophore for Single-Molecule Spectroscopy and Microscopy from $1.4 \mathrm{~K}$ to Room Temperature. J. Phys. Chem. A 1997, 101, 8435-8440.

19 Herrmann, A.; Müllen, K. From Industrial Colorants to Single Photon Sources and Biolabels: The Fascination and Function of Rylene Dyes. Chem. Lett. 2006, 35, 978-985.

20 Kinkhabwala, A.; Yu, Z. F.; Fan, S. H.; Avlasevich, Y.; Müllen, K. Moerner, W. E. Large Single-Molecule Fluorescence Enhancements Produced by a Bowtie Nanoantenna. Nat. Photonics 2009, 3, 654-657.

21 Brinks, D.; Stefani, F. D.; Kulzer, F.; Hildner, R.; Taminiau, T. H.; Avlasevich, Y.; Müllen, K.; Van Hulst, N. F. Visualizing and Controlling Vibrational Wave Packets of Single Molecules. Nature 2010, 465, 905-908.

22 Kim, H. N.; Puhl, L.; Nolde, F.; Li, C.; Chen, L.; Basché, T.; Müllen, K. Energy Transfer at the Single-Molecule Level: Synthesis of a Donor-Acceptor Dyad from Perylene and Terrylene Diimides, Chem. Eur. J. 2013, 19, 9160-9166.

23 Stottinger, S.; Hinze, G.; Diezemann, G.; Oesterling, I.; Müllen, K.; Basché, T. Impact of Local Compressive Stress on the Optical Transitions of Single Organic Dye Molecules. Nat. Nanotechnol. 2014, 9, 182-186.

24 Hutchison, J. A.; Uji-i, H.; Deres, A.; Vosch, T.; Rocha, S.; Müller, S.; Bastian, A. A.; Enderlein, J.; Nourouzi, H.; Li, C.; et al. A Surface-Bound Molecule that Undergoes Optically Biased Brownian Rotation. Nat. Nanotechnol. 2014, 9, 131136.

25 Räder, H. J.; Nguyen, T. T.T.; Müllen, K. MALDI-TOF Mass Spectrometry of Polyphenylene Dendrimers up to the Megadalton Range. Elucidating Structural Integrity of Macromolecules at Unrivaled High Molecular Weights.

Macromolecules 2014, 47, 1240-1248.

26 Cotlet, M.; Vosch, T.; Habuchi, S.; Weil, T.; Müllen, K.; Hofkens, J.; De Schryver, F. Probing Intramolecular Forster Resonance Energy Transfer in a Naphthaleneimide-Peryleneimide-Terrylenediimide-Based Dendrimer by Ensemble and Single-Molecule Fluorescence Spectroscopy. J. Am. Chem. Soc. 2005, 127, 9760-9768.

27 Nguyen, T. T. T.; Türp, D.; Wang, D. P.; Nölscher, B.; Laquai, F.; Müllen, K. A Fluorescent, Shape-Persistent Dendritic Host with Photoswitchable Guest Encapsulation and Intramolecular Energy Transfer. J. Am. Chem. Soc. 2011, 133, 11194-11204.

28 Lubczyk, D.; Grill, M.; Baumgarten, M.; Waldvogel, S. R.; Müllen, K. ScaffoldOptimized Dendrimers for the Detection of the Triacetone Triperoxide Explosive Using Quartz Crystal Microbalances. Chem. Plus. Chem. 2012, 77, 102-105.

29 Nguyen, T. T. T.; Türp, D.; Wagner, M.; Müllen, K. Photoswitchable Conductivity in a Rigidly Dendronized Salt. Angew. Chem. Int. Ed. 2013, 52, 669-673.

30 Moritz, R.; Zardalidis, G.; Butt, H.-J.; Wagner, M.; Müllen, K.; Floudas, G. Ion Size Approaching the Bjerrum Length in Solvents of Low Polarity by Dendritic Encapsulation. Macromolecules 2014, 47, 191-196.

31 Clar, E. Polycyclic Hydrocarbons. Academic Press. London, 1964.

32 Clar, E. The Aromatic Sextet. Wiley. London, 1972.

33 Stabel, A.; Herwig, P.; Müllen, K.; Rabe, J. P. Diode-Like Current-Voltage Curves for a Single Molecule- Tunneling Spectroscopy with Submolecular Resolution of 
an Alkylated, Pericondensed Hexabenzocoronene. Angew. Chem. Int. Ed. 1995, 34, 1609-1611.

34 Samori, P. Scanning Probe Microscopies Beyond Imaging. J. Mater. Chem. 2004, 14, 1353-1366.

35 Watson, M. D.; Fechtenkötter, A.; Müllen, K. Big is Beautiful - 'Aromaticity' Revisited from the Viewpoint of Macromolecular and Supramolecular Benzene Chemistry. Chem. Rev. 2001, 101, 1267-1300.

36 Müllen, K. Graphene as a Target for Polymer synthesis. Adv. Polym. Sci. 2013, 262, 61-92.

37 Mali, K. S.; Adisoejoso, J.; Ghijsens, E.; De Cat, I.; De Feyter, S. Exploring the Complexity of Supramolecular Interactions for Patterning at the Liquid-Solid Interface. Acc. Chem. Res. 2012, 45, 1309-1320.

38 De Feyter, S.; De Schryver, F. C. Self-Assembly at the Liquid/Solid Interface: STM Reveals. J. Phys. Chem. B 2005, 109, 4290-4302.

39 Tan, Y. Z.; Yang, B.; Parvez, K.; Narita, A.; Osella, S.; Beljonne, D.; Feng, X. L.; Müllen, K. Atomically Precise Edge Chlorination of Nanographenes and its Application in Graphene Nanoribbons. Nat. Commun. 2013, 4, 2646.

40 Müllen, K.; Rabe, J. P. Nanographenes as Active Components of SingleMolecule Electronics and How a Scanning Tunneling Microscope Puts Them To Work. Acc. Chem. Res. 2008, 41, 511-520.

41 Diez-Perez, I.; Li, Z.; Hihath, J.; Li, J.; Zhang, C.; Yang, X.; Zang, L.; Dai, Y.; Feng, X. L.; Müllen, K.; et al. Gate-Controlled Electron Transport in Coronenes as a Bottom-Up Approach towards Graphene Transistors. Nat. Commun. 2010, 1, 31.

42 Feng, X.; Marcon, V.; Pisula, W.; Hansen, M. R.; Kirkpatrick, J.; Grozema, F.; Andrienko, D.; Kremer, K.; Müllen, K. Towards High Charge-Carrier Mobilities by Rational Design of the Shape and Periphery of Discotics. Nat. Mater. 2009, 8, 421-426.

43 Britnell, L.; Gorbachev, R. V.; Geim, A. K.; Ponomarenko, L. A. Resonant Tunnelling and Negative Differential Conductance in Graphene Transistors. Nat. Commun. 2013, 4, 1794.

44 Pisula, W.; Feng, X. L.; Müllen, K. Charge-Carrier Transporting Graphene-Type Molecules. Chem. Mater. 2011, 23, 554-567.

45 Chen, D.; Tang, L.; Li, J. Graphene-Based Materials in Electrochemistry. Chem. Soc. Rev. 2010, 39, 3157-3180.

46 Osella, S.; Narita, A.; Schwab, M. G.; Hernandez, Y.; Feng, X. L.; Müllen, K.; Beljonne, D. Graphene Nanoribbons as Low Band Gap Donor Materials for Organic Photovoltaics: Quantum Chemical Aided Design. ACS Nano 2012, 6, 5539-5548.

47 Jiao, L. Y.; Zhang, L.; Wang, X. R.; Diankov, G.; Dai, H. J. Narrow Graphene Nanoribbons from Carbon Nanotubes. Nature 2009, 458, 877-880.

48 Chmil, K.; Scherf, U. A Simple 2-Step Synthesis of a Novel, Fully Aromatic Ladder-Type Polymer. Makromol. Chem. Rapid Commun. 1993, 14, 217-222.

49 Narita, A.; Feng, X. L.; Hernandez, Y.; Jensen, S. A.; Bonn, M.; Yang, H.; Verzhbitskiy, I. A.; Casiraghi, C.; Hansen, M. R.; Koch, A. H. R.; et al. Synthesis of Structurally Well-Defined and Liquid-Phase-Processable Graphene Nanoribbons. Nat. Chem. 2014, 6, 126-132.

50 Abbas, A. N.; Liu, G.; Narita, A.; Orosco, M.; Feng, X. L.; Müllen, K.; Zhou, C. W. Deposition, Characterization, and Thin-Film-Based Chemical Sensing of Ultralong Chemically Synthesized Graphene Nanoribbons. J. Am. Chem. Soc. 2014, 136, 7555-7558. 
51 Lin, Y.-M.; Dimitrakopoulos, C.; Jenkins, K. A.; Farmer, D. B.; Chiu, H.-Y.; Grill, A.; Avouris, P. 100-GHz Transistors from Wafer-Scale Epitaxial Graphene. Science 2010, 327, 662-662.

52 Jensen, S. A.; Ulbricht, R.; Narita, A.; Feng, X. L.; Müllen, K.; Hertel, T.; Turchinovich, D.; Bonn, M. Ultrafast Photoconductivity of Graphene Nanoribbons and Carbon Nanotubes. Nano Lett. 2013, 13, 5925-5930.

53 Rader, H. J.; Rouhanipour, A.; Talarico, A. M.; Palermo, V.; Samori, P.; Müllen, K. Processing of Giant Graphene Molecules by Soft-Landing Mass Spectrometry. Nat. Mater. 2006, 5, 276-280.

54 Treier, M.; Pignedoli, C. A.; Laino, T.; Rieger, R.; Müllen, K.; Passerone, D.; Fasel, R. Surface-Assisted Cyclodehydrogenation Provides a Synthetic Route Towards Easily Processable and Chemically Tailored Nanographenes. Nat. Chem. 2011, 3, 61-67.

55 Lafferentz, L.; Eberhardt, V.; Dri, C.; Africh, C.; Comelli, G.; Esch, F.; Hecht, S.; Grill, L. Controlling On-Surface Polymerization by Hierarchical and SubstrateDirected Growth. Nat. Chem. 2012, 4, 215-220.

56 Chen, Y.-C.; de Oteyza, D. G.; Pedramrazi, Z.; Chen, C.; Fischer, F. R.; Crommie, M. F. Tuning the Band Gap of Graphene Nanoribbons Synthesized from Molecular Precursors. ACS Nano, 2013, 7, 6123-6128.

57 Cai, J. M.; Ruffieux, P.; Jaafar, R.; Bieri, M.; Braun, T.; Blankenburg, S.; Muoth, M.; Seitsonen, A. P.; Saleh, M.; Feng, X. L.; et al. Atomically Precise bottom-up Fabrication of Graphene Nanoribbons. Nature 2010, 466, 470-473.

58 Blankenburg, S.; Bieri, M.; Fasel, R.; Müllen, K.; Pignedoli, C. A.; Passerone, D. Porous Graphene as an Atmospheric Nanofilter. Small 2010, 6, 2266-2271.

59 Palma, C-A.; Samori, P. Blueprinting Macromolecular Electronics. Nat. Chem. 2011, 3, 431-435.

60 Orgiu, E.; Samori, P. 25th Anniversary Article: Organic Electronics Marries Photochromism: Generation of Multifunctional Interfaces, Materials, and Devices. Adv. Mater. 2014, 26, 1827-1845.

61 Samori, P. Exploring Supramolecular Interactions and Architectures by Scanning Force Microscopies. Chem. Soc. Rev. 2005, 34, 551-561.

62 Blankenburg, S.; Cai, J. M.; Ruffieux, P.; Jaafar, R.; Passerone, D.; Feng, X. L.; Müllen, K.; Fasel, R.; Pignedoli, C. A. Intraribbon Heterojunction Formation in Ultranarrow Graphene Nanoribbons. ACS Nano 2012, 6, 2020-2025.

63 Talirz, L.; Söde, H.; Cai, J.; Ruffieux, P.; Blankenburg, S.; Jafaar, R.; Berger, R.; Feng, X. L.; Müllen, K.; Passerone, D.; et al. Termini of Bottom-Up Fabricated Graphene Nanoribbons, J. Am. Chem. Soc. 2013, 135, 2060-2063.

64 Zagranyarski, Y.; Chen, L.; Jänsch, C.; Gessner, T.; Li, C.; Müllen, K. Toward Perylene Dyes by the Hundsdiecker Reaction. Org. Lett. 2014, 16, 2814-2817.

\section{Suggested Pull Quotes}

Polyphenylene dendrimers create a unique functional nanoenvironment by way of a perfect covalent design.

It is a unique feature of our graphene nanoribbon fabrication, both in solution and on surfaces, that their geometric and electronic structures can be tuned by the nature of the monomeric building blocks. 
1

2

3

4

5

6

7

8

9

10

11

12

13

14

15

16

17

18

19

20

21

22

23

24

25

26

27

28

29

30

31

32

33

34

35

36

37

38

39

40

41

42

43

44

45

46

47

48

49

50

51

52

53

54

55

56

57

58

59

60

Nanoscience must go beyond the catalogue of commercial compounds to make use of our collective chemical imagination. 